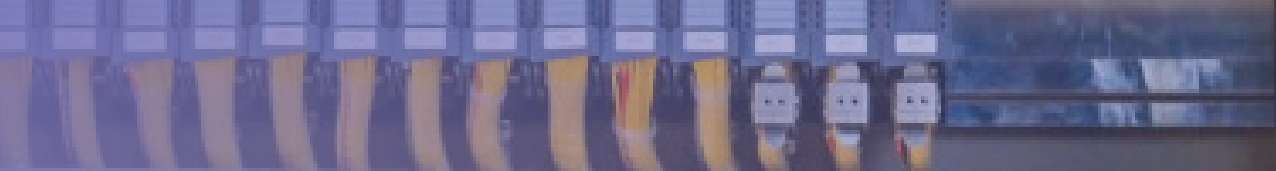

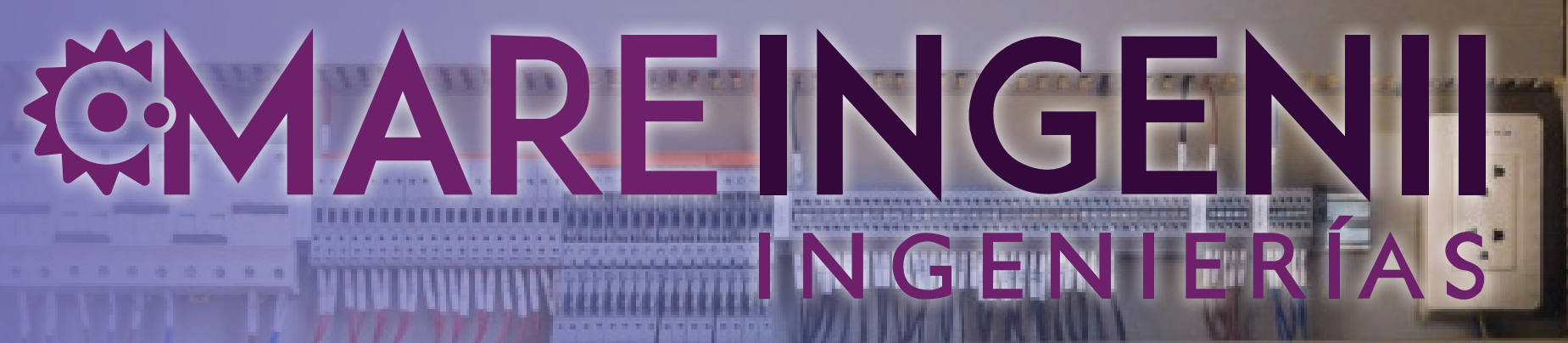

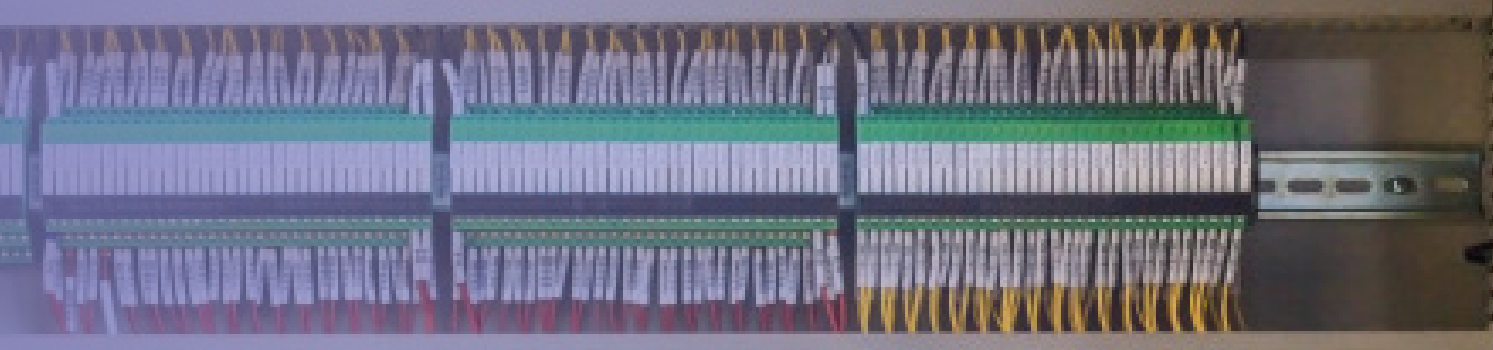

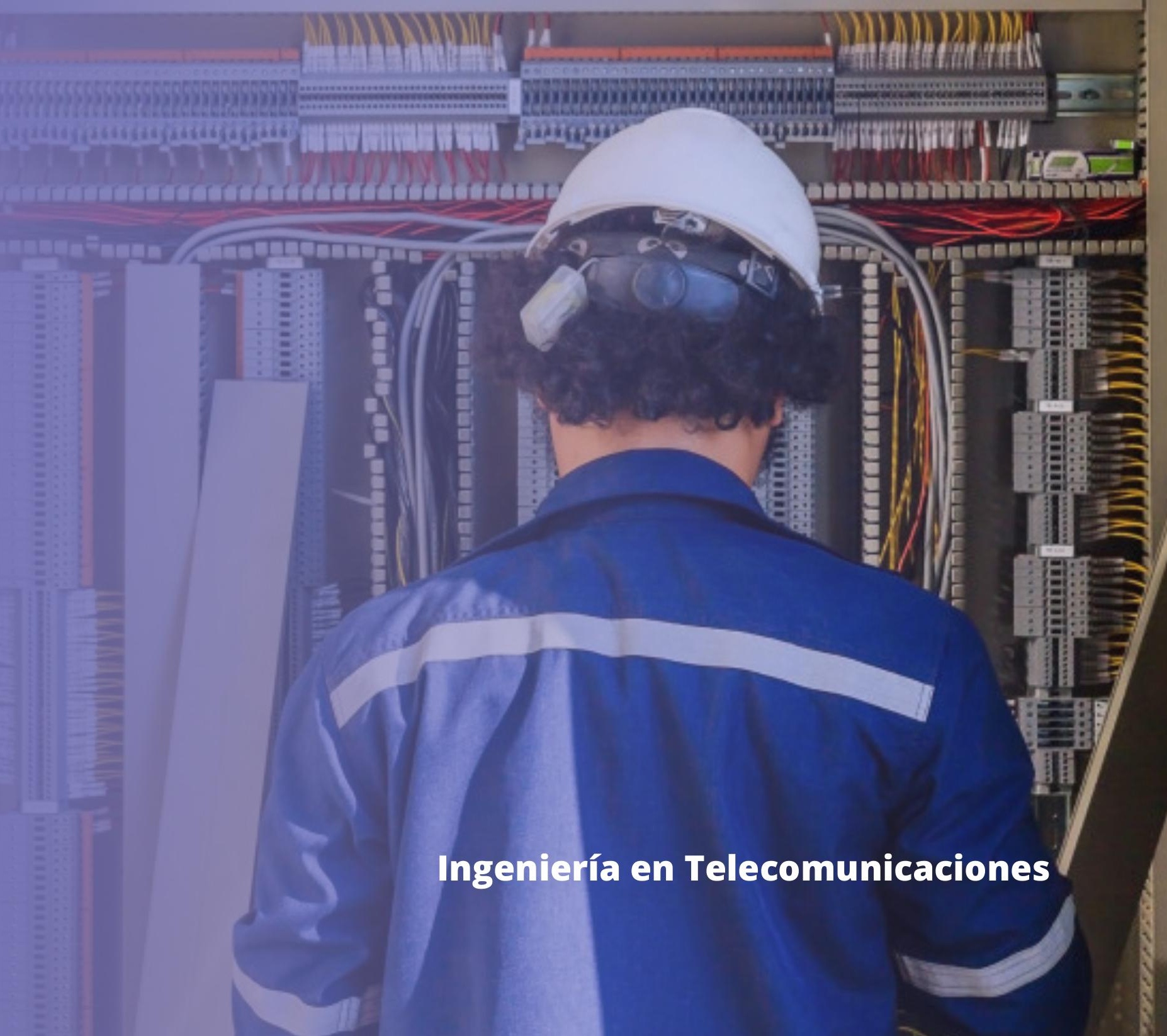




\title{
Tecnología internet of things (IoT) y el big data
}

\section{Internet of Thing (IoT) technology and the big data}

\author{
Wilmar Ferney López Garzón; Juan Camilo Cárdenas López² \\ ${ }^{1}$ Fundación Universitaria San Mateo. Correo electrónico: wflopez@funsanmateo.edu.co \\ 2Fundación Universitaria San Mateo. Correo electrónico: jccardenas@funsanmateo.edu.co
}

Como citar: López Garzón, W; Cárdenas López, J. 2019. Tecnología loT (Internet of Things) y El Big Data. Mare Ingenii. Ingenierías 1(1). Disponible en http://cipres.sanmateo.edu.co/index.php/mi

Recibido: Junio 19 de 2019

Aceptado: Septiembre 11 de 2019

\section{RESUMEN}

Internet of Things (IOT) es la consolidación a través de una red de redes que alberga una gran cantidad de dispositivos. Puede interconectar todos aquellos con interfaces de conexión simples, como electrodomésticos, dispositivos, objetos e incluso torres de construcción con capacidades de comunicación. Todo esto basado en la idea de hacer la vida más cómoda y poder ignorar cosas simples que con una información de variación de datos (big data o macrodatos) pueden controlarse como la temperatura en nuestros hogares, dónde y cuándo usan dispositivos como su vehículo, televisión e incluso la violación del sistema de protección de su hogar. Pero solo en la última década se ha convertido en una realidad a gran escala en la que uno de los inversores y las potencias mundiales ha dedicado un esfuerzo para hacerlo posible ahora. Así, el objetivo de este artículo es identificar las potencialidades del loT y el big data en la situación mundial actual.

Palabras Clave: internet de las cosas; macrodatos; red; telemedicina; seguridad informática; movilidad.

\begin{abstract}
Internet of Things (IOT) is the consolidation, through a network of networks, that harbor a large number of devices. It can interconnect all those with simple connection interfaces such as appliances, devices, objects and even construction towers with communication capabilities. All this based on the idea of making life more comfortable and being able to ignore simple things that with data variation information (big data) can be controlled such as the temperature in our homes, where and when they use devices such as their vehicle, television and even the violation of the home protection system. But only in the last decade has it become a reality on a large scale in which one of the investors and world powers has dedicated an effort to make it possible now. Thus, the objective of this article is to identify the potential of IoT and big data in the current world situation.
\end{abstract}

Keywords: internet of things; big data; network; telehealth; computer security; movilidad. 


\section{INTRODUCCIÓN}

Como entendemos el curso de la vida, está dando pasos de cambio muy importantes, desde cualquier punto que se mire actualmente. Si no nos acoplamos bien a ello, el proceso de aceptación va a ser un poco sinuoso. Esto se debe a la revolución tecnológica que durante los últimos años ha dado grandes cambios. Uno de ellos es el que queremos presentar: el Internet of Things (IOT).

Este esquema, que comenzó sobre el nuevo milenio pero ha tomado mucha fuerza, ha generado un amplio seguimiento de principios de la idea IoT. Dado al sostenimiento por la tecnología Wireless tal y como las conocemos. IoT es la consolidación a través de una red de redes la cual aloja una gran cantidad de dispositivos. Puede interconectar a todos aquellos con interfaces de conexión simples tales como electrodomésticos, dispositivos, objetos y hasta torres de edificios con capacidad de comunicación.

Debido a la cantidad de información que se recolecta por cada dispositivo comunicado en la nube a nivel mundial, es de suma importancia tener una serie de controles para manejar dicha información, por ende la terminología big data, o macrodatos en español. Pero ¿qué es el big data? Es una cantidad voluminosa de datos de diversas características y de diferentes fuentes. Así, para mantener un orden no tan riguroso pero muy asequible, se toma esta información para procesarla mediante esquemas clasificatorios como estructurados, semiestructurados o no estructurados. Dicha clasificación está enfocada en recolectar, analizar e imprimir los resultados obtenidos. Es decir, forja un sistema tan robusto que puede alcanzar un nivel que determine qué necesidad tiene o no un objeto, una sociedad y, en más detalle, una persona.

El big data, como otros enfoques de la tecnología moderna, también está en auge y en simpatía con el mundo actual. Grandes empresas y personal calificado ven en el big data un potencial jamás antes visto por la sociedad moderna. Esta proyección, definida a continuación, ya es una realidad. En nuestra visita al congreso internacional en ingeniería electrónica, eléctrica y de sistemas (INTERCON), realizado en Lima, Perú en el año 2018, los exponentes invitados, compartieron con nosotros este gran crecimiento y foco de cambio en los diversos países desarrollados. Es tanto así que científicos, en España, han implementado el
IoT con el Big data para analizar sus pacientes y tener un mejor control de su salud, calidad de vida y mejoramiento de sus actividades curriculares. Lo anterior, ayudados mediante un conjunto de acciones de comunicación, tanto de hardware como del software que alimentan sus bases de datos.

\section{¿QUÉ ES IOT?}

IoT es la interconexión digital de objetos cotidianos con internet. Permite el cambio automático de información con otros dispositivos o centros de control sin intervención humana, capturando gran cantidad de información clave sobre uso y rendimiento. Así, facilita la monitorización y operación, creando experiencias únicas y oportunidades inéditas para personas empresas y ciudades.

Además, genera eficiencias operativas gracias a la obtención de información en tiempo real sobre el estado de los productos o servicios. También forja capacidades de procesamientos propios que le permiten a los dispositivos ganar cierto grado de 'inteligencia'. La influencia de la palabra IOT fue inscrita en el año 1999, por el profesor Kevin Ashtonn del Massachusetts Institute of Technology (MIT). En aquel entonces, la utilizó de forma pública para definir dicho aspecto importante sobre la conexión del todo [1].

\section{HISTORIA}

La historia del IoT ha tomado fuerza en los últimos años y hay un amplio seguimiento a principios de la década 2000. La idea de IoT de Ashton estaba centrada en el uso de la identificación por radiofrecuencia (RFID) con el fin de interconectar dispositivos entre sí. Por supuesto, el concepto de Ashton de un IOT basado en RFID no era sorprendente en ese momento. En 1999, las redes inalámbricas tal como las conocemos hoy todavía estaban en su infancia y las redes celulares aún no habían cambiado a una configuración totalmente basada en una dirección IP.

En esas condiciones, mencionadas anteriormente, hubiera sido mucho más difícil concebir una IoT en la que todos los dispositivos tuvieran direcciones IP únicas. Además, en ausencia de IPv6, no había suficientes direcciones IP para que todos los dispositivos se conectarán a Internet. Como RFID 
no habría requerido direcciones IP, o conectividad directa a internet para cada dispositivo, hubiera parecido una solución mucho más barata y más factible.

Entre los años 2000 y 2008 la alianza IPSO se formó como una colaboración en la industria, promoviendo dispositivos conectados. Esta fue la señal para que grandes empresas comenzarán a trabajar en la tecnología, para empezar a implementar el IOT en entornos de producción que innovaran en objetos inteligentes, para los nuevos dominios de aplicación y ayudar a facilitar el crecimiento en la loT. Lo anterior permitió poder ser implementado en las industrias como energía inteligente, ciudades inteligentes, atención médica, automatización en el hogar, control de procesos y automatización de edificios.

Por último, la nube ha dado un papel muy crucial en el desempeño para hacer posible el moderno IOT. Su desarrollo proporciona un bajo costo y siempre está en funcionamiento. Con sus diversos procesos, una infraestructura gratuita barata y altamente disponible que nos facilita las descargas, almacenamientos y demás trabajos que se pueden hacer en nuestros dispositivos [1].

Por último, la nube ha tenido un papel crucial en el desempeño para hacer posible el moderno loT. Su desarrollo proporciona un bajo costo.

\section{DESAFÍOS DEL IOT}

Uno de los grandes desafíos para el loT es el de la banda infinita o mayor ancho de banda en las infraestructuras de red. En el momento en que tengamos más dispositivos, mayor será el tráfico que debemos manejar con la red actual. Esto seguirá siendo un factor muy complicado para el crecimiento del loT [2].

La seguridad y la privacidad también son grandes inconvenientes. Los dispositivos relacionados con el IoT, tienen un alto grado de vulnerabilidad. Estos dispositivos recopilan información personal como lo es el número de contacto, los nombres, los correos, la dirección de residencia, el número de cuentas bancarias, entre otros. Después de la cadena interminable de divulgaciones, sobre las brechas de datos más importantes, los consumidores son cautelosos de colocar demasiados datos personales en nubes públicas o privadas, con buenas razones. Los proveedores de IoT deberán resolver estos problemas de seguridad antes de que los dispositivos IOT alcancen su máximo potencial [3].

\section{APLICACIONES DEL IOT}

El apalancamiento que ha tenido la tecnología en diversos sectores de la industria ha sido un factor determinante para que el IOT pueda ingresar con gran facilidad y así demostrar la importancia que tendrá hacia el futuro. Esto quiere decir que las empresas y los dispositivos personales serán más eficientes y competitivos para todos los negocios del mundo actual. Por ende, se relacionan las mejores aplicaciones del IoT en el mercado a futuro. Estas son:

- Edificios inteligentes: es una de las propuestas más importantes que tiene el sector productivo. La digitalización de estos edificios se basará en la transmisión de información de manera constante de todas las disciplinas integradas a este modelo esquemático de arquitectura. Es decir, indicará de manera constante el estado del clima y hasta su sistema de protección contra intrusos o personal no autorizado [3].

- Ciudades conectadas: el IOT, en su esquema general, busca que las ciudades también tengan una forma amena y adecuada de comunicarse entre ellas. Esto, con el fin de tener aprovechamiento del estado de las carreteras viales, el estado del tráfico, la estimación de llegada de una ambulancia en una situación de urgencia, entre otros. Con ello se analizan los resultados para garantizar una calidad de vida mucho mejor.

- Movilidad y Smart grids: en la movilidad, y en la red de energía eléctrica, también es un campo que el loT está incursionando con gran prospecto y concordancia, debido a su gran realidad y sostenibilidad en el mercado actual. La tecnología del IOT distribuirá la energía y las estaciones de carga. De forma tal, el equilibrio energético tendrá un impacto mínimo para el planeta y reducirá hasta en 70\% la contaminación lumínica.

En el enfoque automotriz estos aprovecharán al máximo sus "datos" para gestionar toda la infraestructura que permite la recarga de vehículos eléctricos. Otro aspecto por resaltar, está en el hecho de que cada vehículo tendrá la capacidad de comunicarse con su propietaria informando el estado de las vías, notificar sobre el tránsito, 
indicaciones de sitios sugeridos, etc. Lo anterior revolucionará el estado de nuestra vida como la conocemos. Además, estos dos (2) enfoques tendrán un sistema basado en la tecnología cloud capaz de proporcionar información y servicios a los consumidores y a los agentes comerciales e industriales que participan en la producción, distribución, transporte y comercialización de la energía [3].

- Ciberseguridad: en esta era de la tecnología IOT está generando una revolución de conectividad tanto de electrodomésticos como dispositivos móviles, autos, entre otros, que cuentan con esta capacidad de conectarse a internet con gran facilidad. Hay que preguntarse ¿los dispositivos, o cualquier elemento con conectividad, están realmente seguros frente a amenanzas en línea (online)? En relación con lo anterior, empresas como Cisco Systems ha investigado mejoras para que la seguridad de la información no se presente una problemática al futuro, y traiga con ellos grandes inconvenientes.

Cabe destacar que las brechas de seguridad estarán a la orden del día. Sin embargo, habrán mecánicos de realización y control para que las personas puedan estar seguras con su información. Además, Cisco Systems, subraya que, para el año 2020, el valor económico del loT estará aproximadamente en 19 billones de dólares, con más de 12 millones de dispositivos conectados a la vez. Por lo tanto, el campo de la ciberseguridad está al alcance de nuestras manos.

- Salud y telemedicina: en el entorno de la salud, el loT está presentando un gran potencial que para los pacientes y el personal calificado, como médicos, especialistas, internistas, etc., generará beneficios directos. Su implementación brindará herramientas de administración y análisis de datos recolectados de sus pacientes, mejorando en los diagnósticos y en reducir las enfermedades. Cabe resaltar que en el ámbito económico se reducirá el costo sanitario y se traducirá en un mayor control asistencial para la comunicación médico-paciente.

Ante este panorama, el IoT cobra importancia. Este no es una tendencia que pasará desapercibida. Al contrario, será una transición tan importante para el área de la medicina que transformará la forma de ver la ciencia actual. Además, esta nueva era global y de auge tecnológico también dependerá de los desarrollos mejorados de comunicación y la latencia de las nuevas comunicaciones inalámbricas. Esta transición supondrá muchos retos y talento humano calificado para superar dichas barreras.

\section{BIG DATA}

El Big data, o macrodatos en español, consiste en la gestión y análisis de enormes volúmenes de datos los cuales no pueden ser tratados de manera convencional para el tratamiento procesamiento y análisis de tales datos, resultado de la actividad de cada uno en el internet. El objetivo de estos datos es convertirlo en tomas de decisiones rápidas; esto también es usado en las empresas para evaluar el perfil de los usuarios y cómo se sienten con los servicios que se les venden. Por ejemplo, empresas como Google y Apple presentan anuncios personalizados, dependiendo de los gustos, las compras por internet y las búsquedas realizadas por el usuario [4].

\section{HISTORIA}

Desde que la historia de la raza humana recopila la información a través de la escritura (libros) hasta los centros modernos de datos (discos duros), nunca ha dejado de hacerlo. El crecimiento tecnológico va en aumento lo cual ha provocado un volumen muy alto de datos por lo que son necesarios dispositivos de alta tecnología para almacenar esas grandes cantidades de información. Esta cronología es de la década de 1930, trata de la información y el Big data, creando la necesidad de una nueva organización y modo de guardar la información.

Los académicos han denominado a la expansión de la información, explosión de la información. Tras aparecer por primera vez en el periódico Lawton Constitution, en el año 1964. Allí se hacía referencia a la dificultad que suponía gestionar los volúmenes de información disponibles [4].

\section{APLICACIONES}

- Análisis de operaciones: obtiene visibilidad en tiempo real de operaciones y experiencia de clientes. Hace más dinámico el Programmable Network Access (PNA) para que incremente la eficiencia de operaciones e investiga anomalías, manejo de la infraestructura end-to-end. También evita, de forma preventiva, la degradación y los 
apagones del servicio por medio de un acelerador de datos que permite procesar grandes volúmenes de dichos datos para dar un detallado estado de los mismos. Por ejemplo, los machine data se pueden correlacionar con otros datos como información de clientes o de productos [5].

- Aumentar el almacén de datos o Data Warehouse: la idea es ampliar la estructura ya existente de datos y con el uso del big data, incrementar su valor comercial. El aumento de la data warehouse tiene tres (3) componentes: primero, un núcleo de procesamiento (Pre-Processing Hub) que proporciona un área de montaje o zona de aterrizaje de los datos antes de decidir cuales se incorporan al almacén de datos. Segundo, un descubrimiento de análisis (Discovery/ analytics) el cual da la capacidad de realizar análisis que deberían haberse hecho antes en la data warehouse para así optimizar el almacén de datos y posibilitar nuevos tipos de análisis. Tercero, un almacén de datos de consulta (Query-Able Data Store) que descarga datos que se consultan con poca frecuencia o de una antigüedad considerable del datawarehouse mediante de información, y los almacenan en su espacio de almacenamiento de bajo costo.

- Extensión de seguridad: localiza anomalías y previene ataques. Este tipo de soluciones permite discernir entre cantidades masivas de datos, con relaciones ocultas y patrones de conducta y amenazas a la seguridad. También permite examinar nuevas fuentes y variedades de datos como actividades criminales [5].

- Exploración de los datos: las tres $V$ del Big data (velocidad, volumen y variedad) es el reto al que se enfrentan grandes compañías a la hora de dar los datos para poder mejorar operaciones, tomar decisiones y reducir los riesgos para mantener una información sofisticada.

\section{Ventajas}

Lo anteriormente descrito tiene unos beneficios sorprendentes para un buen análisis de Big data en tiempo real. En primer lugar, permite detectar malas operaciones o problemas financieros de manera rápida. El análisis en tiempo real permitirá a las empresas poder tomar medidas más efectivas para no bajar su producción y tener una ventaja sobre la competencia. Una vez desarrollados buenos perfiles sobre lo que quieren los consumidores; al recopilar toda esta información será valioso para la oportunidad de mejorar las ganancias y el servicio al cliente. El mejor argumento a favor sobre el Big data es que se puede usar para predecir problemas de salud, con medicina de vanguardia. Con este análisis de datos se podrá prevenir enfermedades futuras - comportamientos de ciertas enfermedades congénitas y [6].

\section{Desventajas}

Puede presentar desafíos como en la logística. Las empresas que decidan usar el big data en sus empresas tendrán que modificar sus plataformas y enfoques a medida que los datos que fluyan sean constantes. Esto les exigiría mejores herramientas para realizar análisis sofisticados.

Por otro lado, una de las mayores preocupaciones es la privacidad de los datos. Puede perderse la privacidad de los usuarios y esto se vuelve en un complejo debate. En el caso de los ataques informáticos a las tres (3) V (velocidad, volumen y variedad) para el valor de esta información es necesario contar con un sistema de hackeo de punta para poder proteger estos datos [6].

\section{FUTURO DEL BIG DATA}

Este desarrollo continuo de mejores prácticas en el análisis de datos en tiempo real seguirá siendo una prioridad para la empresas y agencias gubernamentales. Las grandes empresas están de incremento gracias a las iniciativas del big data y esos esfuerzos y dará nuevas capacidades y resultados medibles para una cultura basada en datos. Dichos datos van creciendo mucho más rápido que nunca y se está pensando en convertir grandes cantidades de datos en un valor para cada empresa [5], [7].

\section{CONCLUSIONES}

Las grandes aspectos que tiene IOT a futuro representara un gran avance para el desarrollo de nuevas tecnologías con el fin de proporcionar a cada elemento una conexión y comunicación constante hacia la nube (cloud). Estos enfoques, encaminados al sostenimiento autónomo, requerirán personal calificado que los pueda implementar. Debido a lo anterior, las propuestas que ya tienen muchos 
países a nivel mundial están enfatizados a que generaciones modernas emprendan el camino de la investigación. Una vez se encuentren resultados importantes, se encaminarán en realizar grandes mejoras a elementos existentes los cuales, como se representa en este artículo, han tenido un importante impacto como, por ejemplo, el Smart Home, Smart Car, Smart City etc.

Así, en relación con lo mencionado sobre el IoT, siempre estará ligado o entrelazado con el Big data. Este enfoque de tratamiento de información indicara que los activos, relacionados en el apartado anterior, estarán enviando y recibiendo información de modo online, generando un crecimiento mensurado pero paulatino de diversos sectores de la economía moderna. Esto daría una sostenibilidad importante y potencial a empresas de tecnología y de comunicación.

Es de resaltar que los primeros estudios y mejoras ya se están produciendo en la actualidad en muchos países del mundo. En estos territorios, su mayor afán es alcanzar dichos logros en el año 2020 y así dar a conocer al mundo que los dispositivos convencionales hoy día están sufriendo transformaciones digitales.

Para finalizar, los autores expresan en este artículo que las tendencias que tiene el mundo de las tecnologías y la informática moderna se estarán centralizando en la mejora de dispositivos y elementos ya existentes. Empresas como Lenovo, Apple, Hewlett Packard entre otras, ya están buscando esa forma de innovar e impactar a la sociedad y así trascender en el mundo de la tecnología loT-big data.

\section{LISTA DE REFERENCIAS}

[1] K. Rose, S. Eldridge, L. Chapin, "Orígenes, impulsores y aplicaciones", en La internet de las Cosas - Una breve reseña para entender mejor los problemas y desafíos de un mundo más conectado, 2015, pp. 13-17.

[2] F. A. F. Pérez y J. L. G. Guerra, "Internet de las Cosas", Perspect. s, vol. 10, no 11, pp. 45-49, 2017.

[3] D. Evans, "Internet de las cosas", Cómo la próxima Evol. Internet lo cambia todo. Cisco Internet Bussiness Solut. Group-IBSG, vol. 11, no 1, pp. 4-11, 2011.

[3] F. Telefónica, Smart Cities: un primer paso hacia la Internet de las Cosas, vol. 16. Fundación Telefónica, 2011.
[4] S. Walker, Big data: A revolution that will transform how we live, work, and think. London, United Kingdom: Taylor \& Francis, 2014.

[5] M. Tascón, "Pasado, presente y futuro", Big Data, vol. 95, p. 47, 2013.

[6] L. J. Aguilar, Big Data, Análisis de grandes volúmenes de datos en organizaciones. Ciudad de México, México: Alfaomega Grupo Editor, 2016.

[7] J. Sánchez y M. Cuellar. Análisis de series de tiempo con métodos econométricos para el control de congestión en redes de comunicaciones. Bogotá: Editorial Fundación Universitaria San Mateo, 2018. Recuperado de https://palma.sanmateo. edu.co/ 